\title{
Valsalva computed tomography in visualization of Eustachian tube in patients with patulous Eustachian tube
}

\author{
Suleyman Aksoy ${ }^{1}$, Yavuz Selim Yıldırım², and Işıl Yurdaışık ${ }^{3}$ \\ ${ }^{1}$ Hisar Intercontinental Hospital \\ ${ }^{2}$ Dogus Universitesi \\ ${ }^{3}$ Istinye University
}

January 31, 2021

\begin{abstract}
Abstract Objectives: There is no study in the literature that patients with clinically-proven PET were evaluated by Valsalva CT. We aimed to evaluate the merit of Valsalva computed tomography (CT) in patients who had clinically-proven patulous Eustachian tube (PET). Design: Case-series Setting and participants: We recruited patients with clinically-proven PET. Participants: Main outcome measures: All participants underwent temporal bone CTs while they were performing the Valsalva maneuver in the supine position. Standard axial plane CT images, along with multiplanar reconstruction and 3D Air volume rendering, were used to visualize the ET in its entirety. Results: Three patients ( 2 females, one male) with a total of 6 ears were included in the study. All patients had suggestive symptoms of PET, including aural fullness, aerophony, and autophony and underwent clinical examination and audiologic tests. In all ears, the whole Eustachian tube could be visualized with Valsalva CT. Conclusion: For the first time, we demonstrated the merit of Valsalva CT in visualization of the Eustachian tube in its entirety in patients with clinically diagnosed PET.
\end{abstract}

Valsalva computed tomography in visualization of Eustachian tube in patients with patulous Eustachian tube

\section{Abstract}

Objectives: There is no study in the literature that patients with clinically-proven PET were evaluated by Valsalva CT. We aimed to evaluate the merit of Valsalva computed tomography (CT) in patients who had clinically-proven patulous Eustachian tube (PET).

Design: Case-series

Setting and participants: We recruited patients with clinically-proven PET.

\section{Participants:}

Main outcome measures: All participants underwent temporal bone CTs while they were performing the Valsalva maneuver in the supine position. Standard axial plane CT images, along with multiplanar reconstruction and 3D Air volume rendering, were used to visualize the ET in its entirety.

Results: Three patients ( 2 females, one male) with a total of 6 ears were included in the study. All patients had suggestive symptoms of PET, including aural fullness, aerophony, and autophony and underwent clinical examination and audiologic tests. In all ears, the whole Eustachian tube could be visualized with Valsalva CT.

Conclusion: For the first time, we demonstrated the merit of Valsalva CT in visualization of the Eustachian tube in its entirety in patients with clinically diagnosed PET. 
Keywords : Computed Tomography; Eustachian tube; Patulous Eustachian tube; Valsalva maneuver

Key points;1. Patulous Tuba Eustachii is not clearly seen on routine radiological imaging.

2. Valsalva CT is never performed to diagnose the patulous Tuba Eustachii.

3. CT is commonly used in daily practice of the ENT.

4. Valsalva maneuver can easily be done by patients during CT examination of the patients who have suspicion of patulous Tuba Eustachii.

5. Patulous Tuba Eustachii can easily be seen on Valsalva CT.

\section{Introduction}

The Eustachian tube (ET) is an anatomical structure that extends from the nasopharynx up to the middle ear and is composed of a bony and a cartilaginous part. Its normal function is vital for healthy hearing (1). This epithelial-lined tube has three main functions: (i) equalization of the pressure in the middle ear to the atmospheric pressure. This is important to curb the untoward effects of external pressure fluctuations on the tympanic membrane. (i) clearance of middle ear secretions, (iii) prevention of middle ear from nasopharyngeal secretions containing microorganisms and precluding transmission of one's own speech sounds back to their ear (2).

Eustachian tube dysfunction might be due to either obstruction of the tube or persistent opening of it (3). ET, under physiologic conditions, remains passively closed and only briefly opens in case of swallowing or Valsalva maneuver. When the ET remains permanently open, this condition is called patulous Eustachian tube (PET). Although its prevalence is much less compared with the obstructive pathologies of the ET, PET is associated with annoying symptoms that lower the quality of life of the affected patients. Patients with PET mainly complaint about hearing their own voice (autophony), their breathing (aerophony) or sound of eating or drinking in a disturbing way. Inflicted patients can also report other more nonspecific symptoms such as aural fullness, vertigo, and tinnitus (4).

Due in part to its inaccessible nature, there is not a single reliable test correctly diagnose ET dysfunction. Constellation of the aforementioned symptoms in a patient suggests the presence of PET; however, these symptoms can also be reported by patients with several other disorders. Thus, more objective and reliable tests are needed. Tympanometry is the commonly used office-based test to evaluate the ET function indirectly. This test measures the one-time middle ear pressure. Normal pressures measured with tympanometry does not exclude obstructive ET dysfunction or PET. Breathing-synchronous tympanic membrane movement is accepted as a specific finding of PET but, to diagnose PET, various objective and subjective findings, such as medical history, physical examination and ET function tests, are combined, because there is no single test available to evaluate ET function accurately. Clinical tympanic membrane movement can be carried out by visual observation, continuous impedance recording, or sonotubometry $(5,6)$. However, these tests are not well standardized and limited in cases of intermittent PET or patients who had low acoustic impedance.

Radiologic imaging of the ET is also without its own problems. Currently, there is no single radiologic imaging modality that can provide details of all related structures of the ET per se. The collapsed nature of the ET at rest makes it more difficult to image the entire tube. One can think that this cannot be a problem for a PET patient. However, the ET temporarily closes when the patient lies supine, which is the usual position in sectional imaging modalities (7). Thus, several investigators tried to find some ways to overcome these difficulties, such as CT imaging in a seated patient or positioning the patient in 45 degrees in a standard CT scanner (8-10). However, these modifications are not always technically feasible. One other solution to the imaging problem of PET is performing a Valsalva maneuver during CT scanning. Self-administered pressure increases force the ET to open even in a patient who is lying supine.

\section{Aim and Purpose}

Valsalva CT of temporal bone has been performed in patients with nose related complaints (11) and patients 
who would undergo surgery for chronic ear disease (12). To the best of our knowledge, there is no study in the literature that patients with clinically-proven PET were evaluated by Valsalva CT. Thus, we aimed to evaluate the ability of Valsalva CT in demonstrating patency of ET in its entirety in patients with symptoms strongly suggestive of PET.

\section{Materials and methods}

This was a retrospective case series in which we assessed the feasibility of Valsalva maneuvered computed tomography scanning in the diagnosis of patulous Eustachian tube (PET). All patients assessed for eligibility for inclusion in the study applied with complaints suggestive of PET to the outpatient clinics of otolaryngology head and neck surgery of a private university hospital. Recruited patients were diagnosed with PET based on their symptoms and clinical tests. In these patients, temporal bone computed tomography was performed while patients were in the supine position and again when they were performing a Valsalva maneuver.

Institutional Review Board of XXX University approved the study protocol (2/2020.K-054).

Patients

In general, none of the patients had a structural ear abnormality or prior ear surgical procedure. All patients underwent Eustachian function tests, which revealed that all patients had functional and patent Eustachian tubes. None of the patients had an obstructing nasal pathology such as septum deviation, concha hypertrophy, nasal polyp, or adenoids. During ear examination, special attention was paid to the tympanic membrane movements. Middle ear pressures were measured by tympanometry in the supine position with and without the Valsalva maneuver. Middle ear pressures were found to be more than +10 daPA on the right and the left ear.

Patient-1. Thirty-two-year-old female patient. The patient complained hearing of her own eating or drinking sounds, ringing in the ears, and autophony for the last 2 years. Ear physical examination revealed normal ears. The patient reported an intentional weight loss of $12 \mathrm{~kg}$ during a 3-month period, after which her complaints had started. She was treated for allergic rhinitis that did not alleviate her symptoms. We performed tympanometric and audiologic tests which showed no pathology. The patient underwent temporal bone computed tomography, the result of which was compatible with the patulous Eustachian tube. We recommended that the patient should gain weight.

Patient-2. Thirty-eight-year-old female patient. The patient applied with complaints of hearing her own speaking sounds in an uncomfortable way and intolerance of loud sounds. Ears were found normal in otoscopic examination. The nasal exam was also normal. The patient stated that her symptoms started after she gave birth one year earlier. Tympanometric and audiologic tests revealed normal results. Temporal bone computed tomography showed findings compatible with PET.

Patient-3. Forty-eight-year-old male patient. The main complaints of the patient were hearing his own eating and drinking sounds, tinnitus, and aerophony. The patient has been using a decongestant nasal spray for about 10 years. He reported an increase in his symptoms for the last 6 months. Nasal physical examination showed rhinitis medicamentosa due to continuous decongestant use. Ear examination was normal except for a slightly flask tympanic membrane. Audiologic assessment did not show any abnormalities. Temporal bone computed tomography was carried out.

Valsalva computed tomography procedure

While the patient was in the supine position in the CT machine, we first obtained images (pre-Valsalva CT) in the neutral position. Then the patient was asked to perform Valsalva maneuver as forceful exhalation against a closed nose and images in the axial plane (Valsalva CT) were obtained. Toshiba Aquilion One 320 -detector row 640 -slice dynamic volume CT system $(160 \times 0.5$ volume scanning mode) was used. We made multiplanar reconstruction (MPR) of the images using the workstation to display the whole axis of the Eustachian tube. In order to display the Eustachian tube with its whole axis, we took the nasopharyngeal 
orifice of the ET as the center of the rotation, and axial plane was tilted antero-inferiorly while looking at the sagittal and coronal images until the ET was shown throughout its axis.

We also demonstrated the air tract between the oropharynx and tympanic cavity (patulous Eustachian tube) three-dimensionally by means of the 3D Air Volume rendering method.

Results

Three patients (two females, one male) with a total of 6 ears with PET were included in the study. The ages of the patients were 32,38 , and 46 years. All patients had symptoms suggestive of patulous Eustachian tube, and clinical history, examination and tests confirmed the diagnosis. In all patients, temporal bone CT showed that the distal one-third of the Eustachian tube was closed while the patient was in the (a) supine position (Figures 1a and 1b). In CT images taken during the Valsalva maneuver (Valsalva CT), the Eustachian tube was visualized in its entirety in all scanned ears (Figures $2 \mathrm{a}$ and $2 \mathrm{~b}$ ).

\section{Discussion}

The main finding of this study was that Valsalva CT was effective in demonstrating the whole length of Eustachian tube in patients with suggestive symptoms of patulous Eustachian tube (PET). We could visualize $\mathrm{ET}$ in its entirety in all six ears.

Patulous Eustachian tube is generally diagnosed based on suggestive symptoms, physical examination, and audiologic findings. However, no diagnostic gold standard is currently available. With an attempt to reduce the subjectivity in the diagnosis of PET, several specialized tests, including tubomanometry, sonotubometry, and tubotympanoaerodynamography, have been devised $(13,14)$. However, these methods provide indirect information and cannot provide the anatomic nature of the ET. Computed tomography and magnetic resonance imaging have been used to visualize ET in patients with ET dysfunction. Yoshida et al. reported that standard CT visualized only 13 ETs entirely as open out of 31 PET ears. A systematic review evaluating the value of the imaging modalities in PET revealed that $\mathrm{CT}$ and MRI provided detailed anatomic information about the ET; nevertheless no single test emerged as a stand-alone diagnostic method in PET (15).

One inherent nature of the ET that is closure of the cartilaginous part of the tube in the recumbent position makes these methods unreliable in the evaluation of PET.

To be able to show the patency status of the ET with cross-sectional imaging modalities, several groups attempted various methods. Yoshida et al. (16) compared the air space lumen between the sitting and recumbent positions in $\mathrm{CT}$ in two PET patients. The size of the airspace lumen was larger during the sitting CT. Kikuchi and colleagues reported the beneficial use of sitting position Valsalva CT in the diagnosis of PET (17). Kawamura and coworkers showed the value of sitting position CT relative to other diagnostic tests in Patients with PET (4). However, sitting position CT is not feasible with standard CT machines. Thus, methods to prevent the collapse of the ET in recumbent positions were sought.

One such method that enables ET to open is the performance of Valsalva maneuver during the CT when the patient is in the supine position. Tarabichi et al. (11) performed Valsalva CT in 38 patients who had nose pathologies but no ear disease. The authors concluded that Valsalva CT could visualize the whole length of the ET tube in $35 \%$ of the ears, whereas in $94 \%$ of the ears, distal one-third of ET could be visualized. Poor Valsalva technique was associated with paradoxical closure of the ET in this study. Another case-control study conducted by the same authors and recruited patients who would undergo surgery for chronic ear diseases found that Valsalva CT could visualize distal one-third of the ears in $96 \%$ of the ears (12). However, to the best of our knowledge, the value of the Valsalva CT in visualization of the entire ET has not been evaluated in patients who had clinically diagnosed PET or patients who had suggestive symptoms for PET yet.

Our results showed that in all 6 ears studied; ET could be visualized entirely by Valsalva CT. We also demonstrated 3D and air-reconstructed images of patent ET. Our findings lend support to previous studies in which Valsalva CT had merit in visualization of the ET in patients who had ear or nose diseases other 
than PET. Our findings extend the prior findings to patients with clinically-proven PET. In all ears, the Valsalva maneuver could preclude the collapse of the distal part of the ET.

Some limitations of the present study deserve mention. First, ours was a preliminary study with only 6 ears. The number of the patients in this study is relatively small however, all of the patients were selected to represent clinical characteristics of PET. None of them had chronic ear and/or nose disease other than aforementioned symptoms. Moreover, patients with relatively severe PET visit our Hospital department to seek treatment.

\section{Conclusion;}

As far as we know, this was the first report of the utilization of supine Valsalva CT in patients with clinically diagnosed PET. It is a clear research need to extend our findings in larger patient samples.

\section{Data availability statement;}

The data that support the findings of this study are available from the corresponding author upon reasonable request.

\section{References:}

1. Bance M, Tysome JR, Smith ME. (2019) Patulous Eustachian tube (PET), a practical overview. World J Otorhinolaryngol Head Neck Surg. 5:137-42.

2. Ars B, Dirckx J. (2016)Eustachian Tube Function. Otolaryngol Clin North Am.49:1121-33.

3. Schilder AG, Bhutta MF, Butler CC, Holy C, Levine LH, Kvaerner KJ, et al. (2015) Eustachian tube dysfunction: consensus statement on definition, types, clinical presentation and diagnosis. Clin Otolaryngol.40:407-11.

4. Kawamura Y, Ikeda R, Kikuchi T, Miyazaki H, Kawase T, Katori Y, et al. (2019) The characteristic of patulous eustachian tube patients diagnosed by the JOS diagnostic criteria. PLoS One.14):e0226908.

5. Schroder S, Lehmann M, Korbmacher D, Sauzet O, Sudhoff H, Ebmeyer J. (2015) Evaluation of tubomanometry as a routine diagnostic tool for chronic obstructive Eustachian tube dysfunction. Clin Otolaryngol.40:691-7.

6. McGrath AP, Michaelides EM. (2011) Use of middle ear immittance testing in the evaluation of patulous eustachian tube. J Am Acad Audiol.22:201-7.

7. Brace MD, Horwich P, Kirkpatrick D, Bance M. (2014) Tympanic membrane manipulation to treat symptoms of patulous eustachian tube. Otol Neurotol.;35:1201-6.

8. Kikuchi T, Oshima T, Hori Y, Kawase T, Kobayashi T. (2009) Three-dimensional computed tomography imaging of the eustachian tube lumen in patients with patulous eustachian tube. ORL J Otorhinolaryngol Relat Spec.71:312-6.

9. Yoshioka S, Naito K, Fujii N, Katada K. (2013) Movement of the Eustachian tube during sniffing in patients with patulous Eustachian tube: evaluation using a 320-row area detector CT scanner. Otol Neurotol.34:87783.

10. Ikeda R, Kikuchi T, Oshima H, Miyazaki H, Hidaka H, Kawase T, et al. (2016) Relationship Between Clinical Test Results and Morphologic Severity Demonstrated by Sitting 3-D CT in Patients With Patulous Eustachian Tube. Otol Neurotol.37:908-13.

11. Tarabichi M, Najmi M. (2015) Visualization of the eustachian tube lumen with Valsalva computed tomography. Laryngoscope.125:724-9.

12. Tarabichi M, Najmi M. (2015)Site of eustachian tube obstruction in chronic ear disease. Laryngoscope.125:2572-5. 
13. Smith ME, Takwoingi Y, Deeks J, Alper C, Bance ML, Bhutta MF, et al. (2018) Eustachian tube dysfunction: A diagnostic accuracy study and proposed diagnostic pathway. PLoS One.13:e0206946.

14. Sudhoff HH, Mueller S. (2018) Treatment of pharyngotympanic tube dysfunction. Auris Nasus Larynx. 45:207-14.

15. Smith ME, Scoffings DJ, Tysome JR. (2016) Imaging of the Eustachian tube and its function: a systematic review. Neuroradiology.58:543-56.

16. Yoshida H, Kobayashi T, Morikawa M, Hayashi K, Tsujii H, Sasaki Y. (2003) CT imaging of the patulous eustachian tube-comparison between sitting and recumbent positions. Auris Nasus Larynx.30:135-40.

17. Kikuchi T, Oshima T, Ogura M, Hori Y, Kawase T, Kobayashi T. (2007) Three-dimensional computed tomography imaging in the sitting position for the diagnosis of patulous eustachian tube. Otol Neurotol.28:199203.

\section{Figure Legends}

Figure 1. Appearance of the cartilage part of the Eustachian tube in temporal bone 3D air-reconstruction (1a) and CT with MPR (1b) when the patient was in the supine position.

Figure 2. Appearance of the Eustachian tube in temporal bone CT with 3D air-reconstruction (2a) and MPR (2b) when the patient was performing a Valsalva maneuver in the supine position. Note that the Eustachian tube can be seen from the nasopharynx up to the middle ear in its entirety.

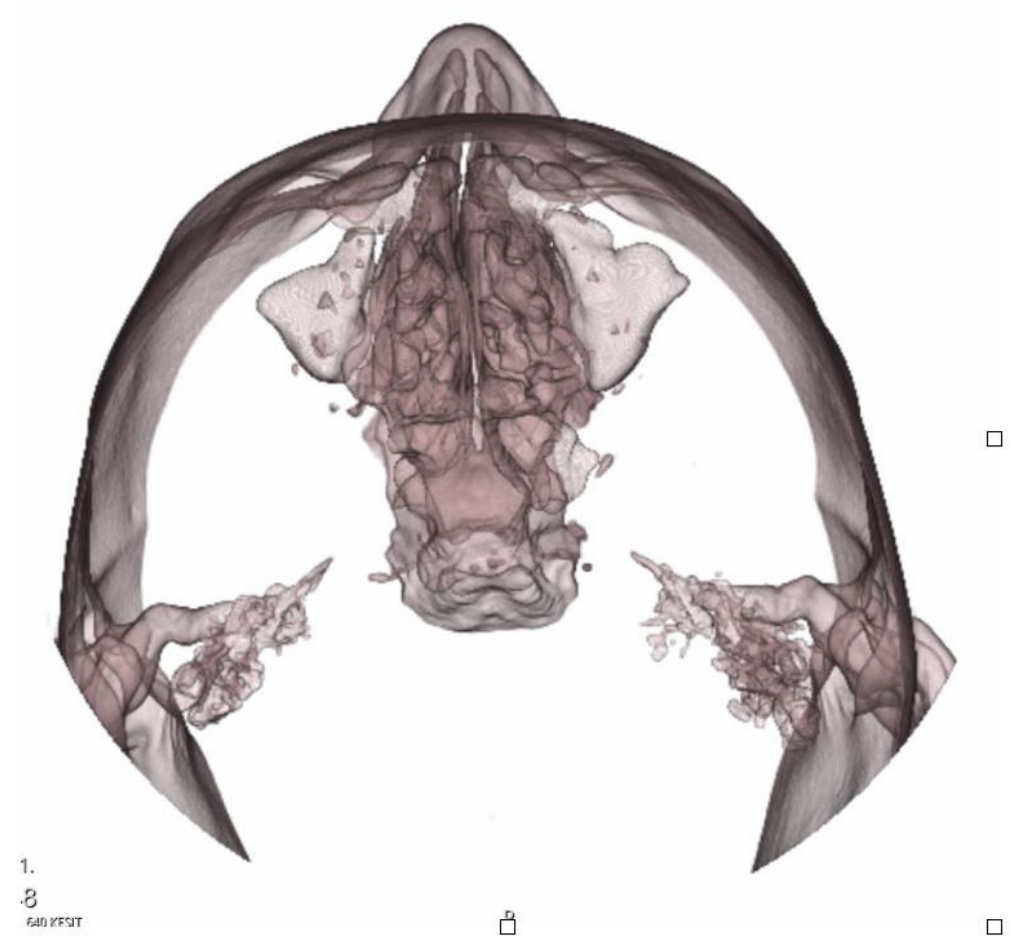




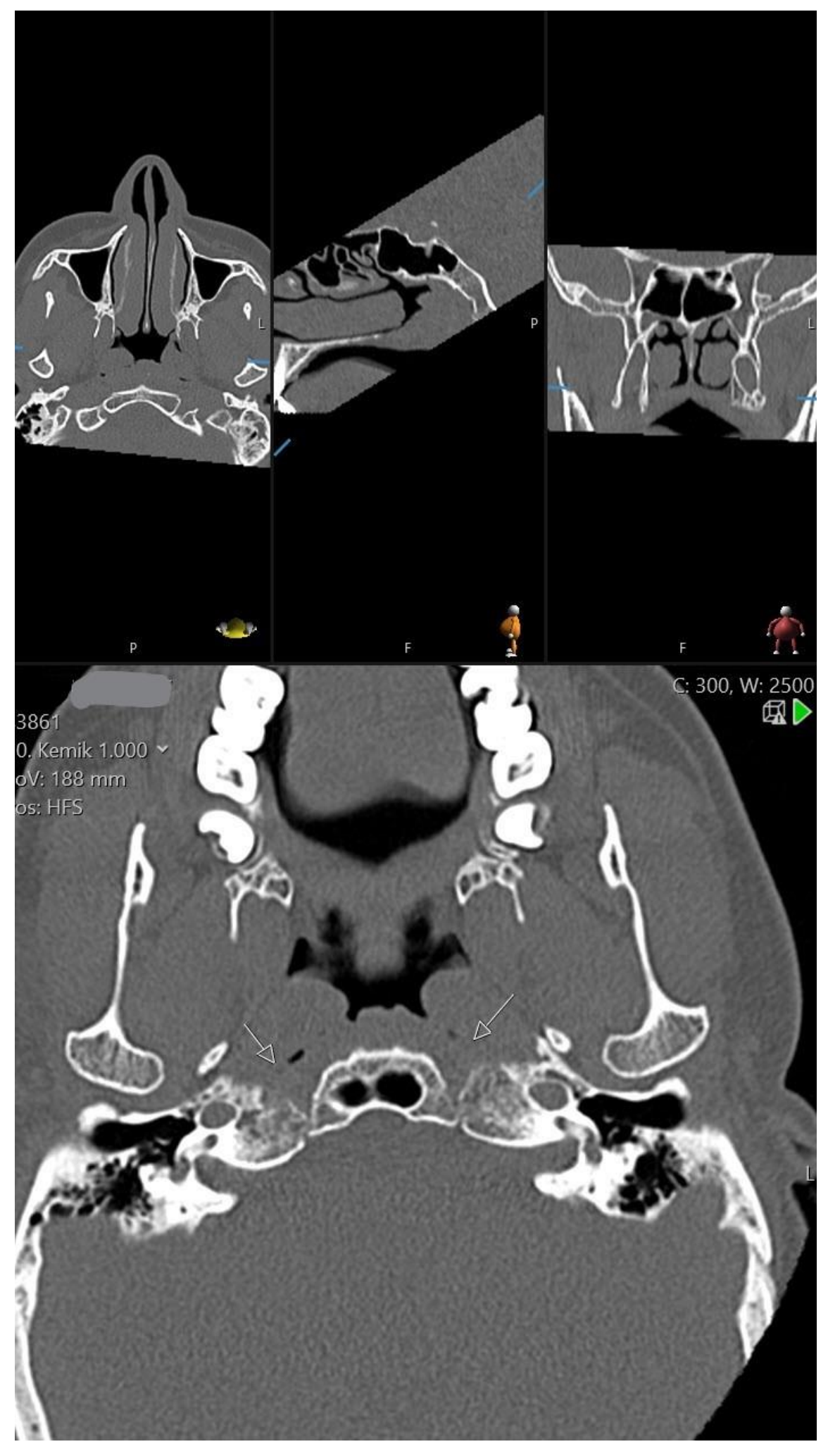




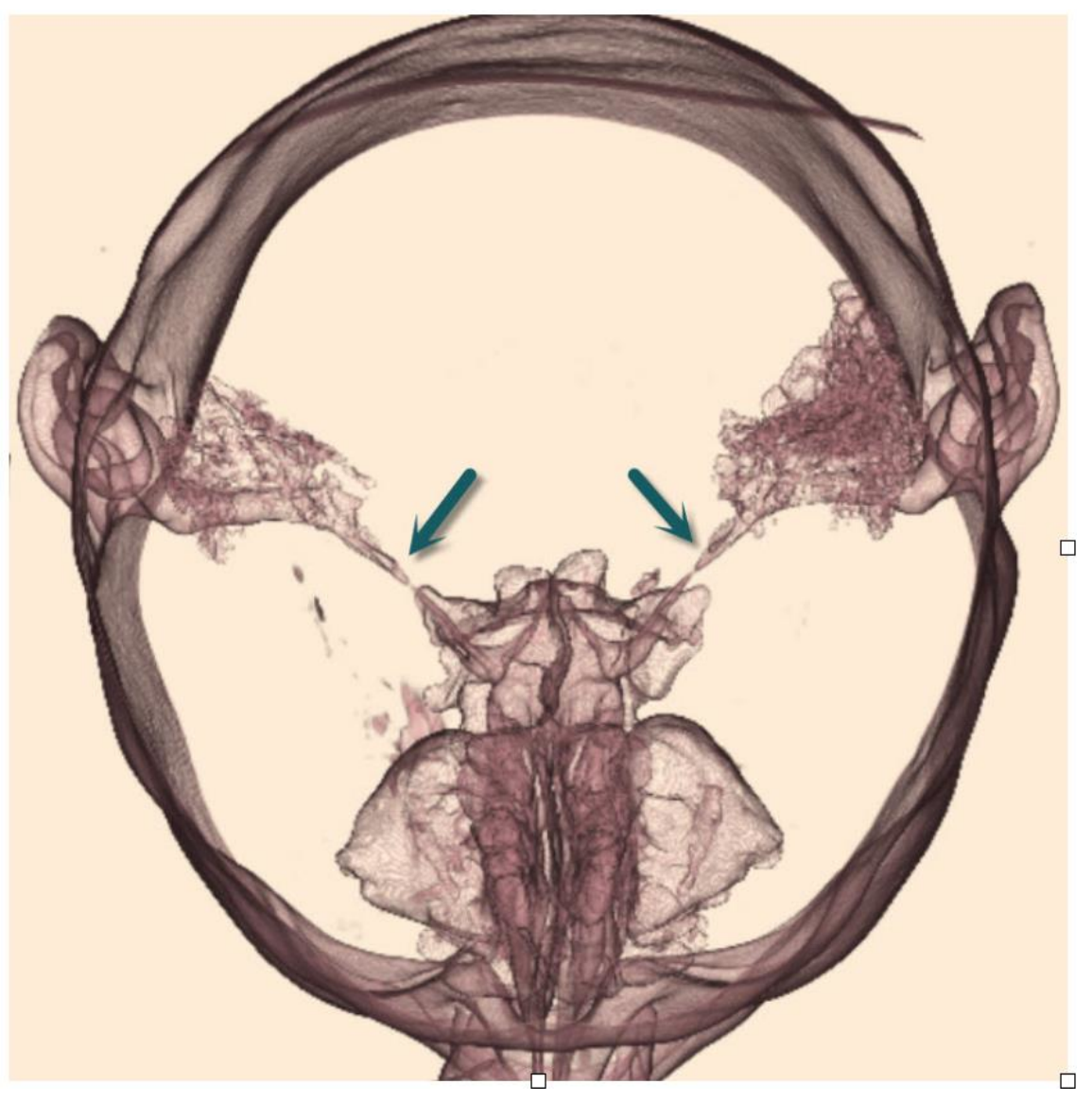




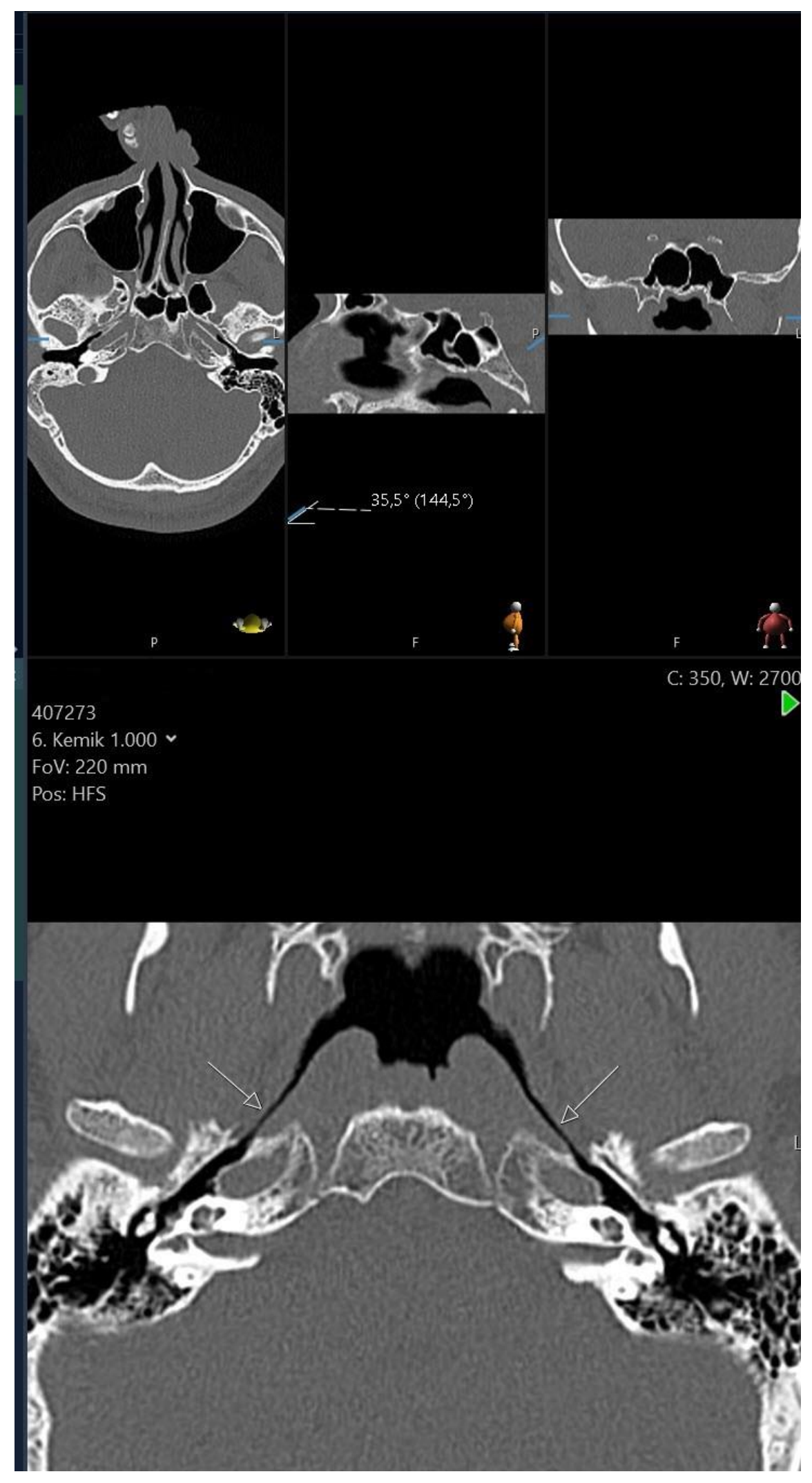

